\title{
Search for signal emission from unresolved point sources with the ANTARES neutrino telescope
}

\section{Rodrigo Gracia Ruiz on behalf of the ANTARES collaboration*}

APC, Université Paris Diderot, CNRS/IN2P3, CEA/Irfu, Observatoire de Paris, Sorbonne Paris Cité, 10 rue Alice Domon et Léonie Duquet, 7205 Paris Cedex 13, France

E-mail: rgracia@in2p3.fr

\begin{abstract}
A two point correlation analysis is used to search for inhomogeneities in the arrival directions of the high energy muon neutrino candidates detected by the ANTARES neutrino telescope. This approach is complementary to a point source likelihood-based search, which is mainly sensitive to point like sources and not to collective effects. We present the results of a search based on this two-point correlation method performed on ANTARES 2007-2012 data, providing constraints on models of a population of point sources too faint to be detected by the searches for point like sources.
\end{abstract}

The 34th International Cosmic Ray Conference,

30 July- 6 August, 2015

The Hague, The Netherlands

${ }^{*}$ Speaker. 


\section{Introduction}

The origin of cosmic rays (CR) is still an open question that can only be addressed by identifying their sources and the physical mechanisms by which they are accelerated up to energies of the order of $\sim 10^{20} \mathrm{eV}$. The magnetic fields in the galactic and intergalactic space deflect the CRs during their propagation, making it difficult to resolve their source's positions by measuring their arrival direction. Neutrinos are believed to be produced in hadronic processes in the CR accelerators. The fact that they are electrically neutral and weakly interacting particles make of neutrinos good candidates to determine unambiguously the position of the CR accelerators.

Neutrino telescopes aim at detecting the Cherenkov light emitted by charged leptons resulting from the interaction of astrophysical neutrinos with the matter surrounding the instrumented volume. The good angular resolution (below $0.5^{\circ}$ ) achieved with the ANTARES neutrino telescope for muon tracks allows for the search of small scale anisotropies (eg point sources) as well as large scale structures. In the following a model independent search is presented based on a modified two point correlation function. The results are interpreted in terms of upper limits on the population of unresolved point-like sources.

\subsection{Motivation}

The interaction of high energy cosmic rays with the Earth's atmosphere induces air showers in which among other particles, muons and neutrinos are present. These so called atmospheric muons and atmospheric neutrinos constitute the two main sources of background for the ANTARES detector. Given that the Earth is opaque to all particles with the exception of neutrinos, because they interact weakly with matter, the atmospheric muon background can be reduced by selecting only those events that are reconstructed with an upwards direction with respect to the ANTARES neutrino telescope. Nevertheless, some muon tracks coming from above can be reconstructed as up going. The amount of wrongly reconstructed muons can be reduced by means of quality cuts in the muon tracks reconstruction parameters.

Atmospheric neutrinos can traverse the Earth and produce muon tracks that will remain as an irreducible source of background.

The challenge of the statistical analyses carried out within the ANTARES collaboration is to unmask those events with astrophysical origin, hidden within a background dominated ensemble of isotropically reconstructed events. One way of looking for an astrophysical signal, is to look for clustering in the arrival directions of the reconstructed events. The autocorrelation analysis is a way of looking for spatial clustering in discrete data ensembles. An improved autocorrelation analysis was presented in [2] and applied to the neutrino candidates detected by the ANTARES neutrino telescope during its first three years of data taking. In the present analysis the method is applied to five years of data and used to search for a signal coming from sources that are too faint to be detected by other statistical analyses such as the ones relying on a likelihood-based method [3]. In absence of such a signal we will set upper limits on the neutrino fluxes. 


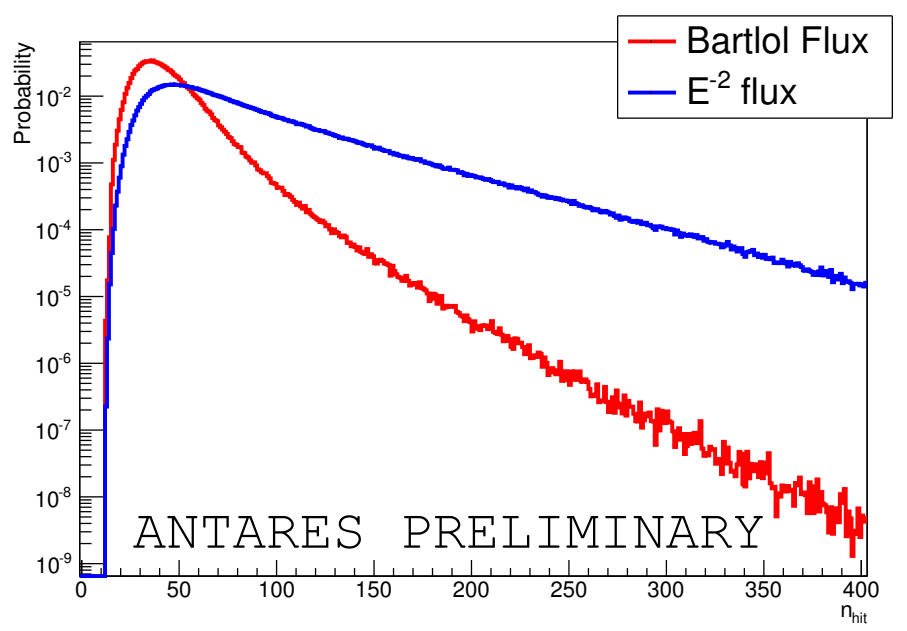

Figure 1: Distributions of the $n_{\text {hit }}$ energy estimator for simulated atmospheric neutrinos, following a Bartol flux $\left(\sim E^{-3.7}\right)$, and simulated cosmic neutrinos following an $E^{-2}$ spectrum.

\section{The autocorrelation analysis}

\subsection{The method}

The autocorrelation analysis allows to find inhomogeneities within a discrete data set by studying the two point correlation distribution, which is defined as the distribution of the number of pairs of events as a function of their mutual angular distance $\Delta \Omega$. As it was shown in [2] and [4], weights based on some energy estimator $\bar{E}$ can be applied to the events in order to discriminate between astrophysical neutrinos, which dominate at lower energies, and cosmic neutrinos, which spectral distribution in energy is harder. This behaviour is shown in fig. 1 for simulated events.

Formally, the cumulative autocorrelation distribution can be defined as

$$
\mathscr{N}_{n_{H i t}}(\Delta \Omega)=\sum_{i=1}^{N} \sum_{j=i+1}^{N} \omega_{i j} \cdot\left[1-H\left(\Delta \Omega_{i j}-\Delta \Omega\right)\right]
$$

where $H$ is the Heaviside step function and $\omega_{i j}=\omega_{i} \cdot \omega_{j}$ are weights assigned to the couple of events $i$ and $j$. Each of the individual weights $\omega_{i}$ is defined as

$$
\omega\left(\bar{E}_{i}\right)=\int_{0}^{\bar{E}_{i}} f(\bar{E}) d \bar{E}
$$

$f(\bar{E})$ is the normalized distribution of the energy estimator, which can be obtained from Monte Carlo simulations. As it is shown in [2], the selected choice for the energy estimator is the number of hits used in the event reconstruction, $n_{\text {hit }}$. Figure (1) shows a comparison between the $n_{\text {hit }}$ distribution for atmospheric and astrophysical neutrinos.

A comparison of the autocorrelation function resulting from measured events with the one corresponding to an isotropic sample will allow to detect possible clusters of events if a significant excess with respect to the isotropy is present in the data. 


\subsection{The ANTARES data set}

For the present analysis, a data set recorded by the ANTARES neutrino telescope between 2007 and 2012 has been used. The sample contains 5243 neutrino candidates that satisfy selection criteria optimized in order to obtain the best average upper limit on the flux of neutrino coming from point like sources and extends the dataset of the previous analysis [2] by about 50\%. These selection criteria consist in a cut on the reconstructed zenith angle $\theta>90$, a cut on the angular uncertainty in the track reconstruction $\beta<1$, and a cut on the reconstruction quality parameter $\Lambda>-5.2$

\subsection{The reference autocorrelation distribution}

The reference autocorrelation distribution has been built as the average of the autocorrelation distributions derived from about $10^{4}$ isotropic data sets. Each of them was obtained by keeping the local coordinates of one neutrino candidate, but assigning it a time randomly selected from another event. This process allows to build an isotropic sky map with the same coverage as the original data set, and in which the non uniformity in the data taking conditions is taken on account.

\subsection{Statistical comparison between the data and the reference distributions}

Fig. 2 shows the cumulative autocorrelation distributions described in section 2.1. The statistical comparison between both distributions is based on an hypothesis test in which the test statistics (TS) will be given by the maximum of a quantity computed for each angular scale, that measures the difference between both distributions:

$$
T S=\max \left\{\left(\frac{\mathscr{N}_{n_{\text {Hit }}^{\text {data }}}^{\text {da }}-\mathscr{N}_{n_{\text {Hit }}^{\text {iso }}}^{\text {is }}}{\sigma}\right)\right\}_{\Delta \Omega_{i}}
$$

where $\sigma$ denotes the standard deviation for the isotropic distribution with respect to its mean.

A distribution of the test statistics for background like ensembles will be built by comparing the autocorrelation distribution of about $10^{4}$ randomized sky maps with the isotropic one. This distribution will be used to compute the p-value as the probability of finding in a background like ensemble, the same value for the test statistics or a higher one than the corresponding to the data.

\subsection{Performance and sensitivity of the method}

The detection power of the autocorrelation method has been previously tested with background sky maps in which some of the events had been substituted by signal events that would have come from a single point source [2]. The results, showed that a dedicated point source search analysis is slightly more efficient in the detection of single point sources than the autocorrelation method, but outperforms it as soon as more than one source is present. Here the detection power of this method is studied using signal coming from populations of point sources that are too faint to be detected by the dedicated point source search.

\subsubsection{Model for sky maps with signal}

In background like sky maps produced as described in section 2.3, a certain proportion of events has been removed from their positions and substituted by signal like events distributed in a 


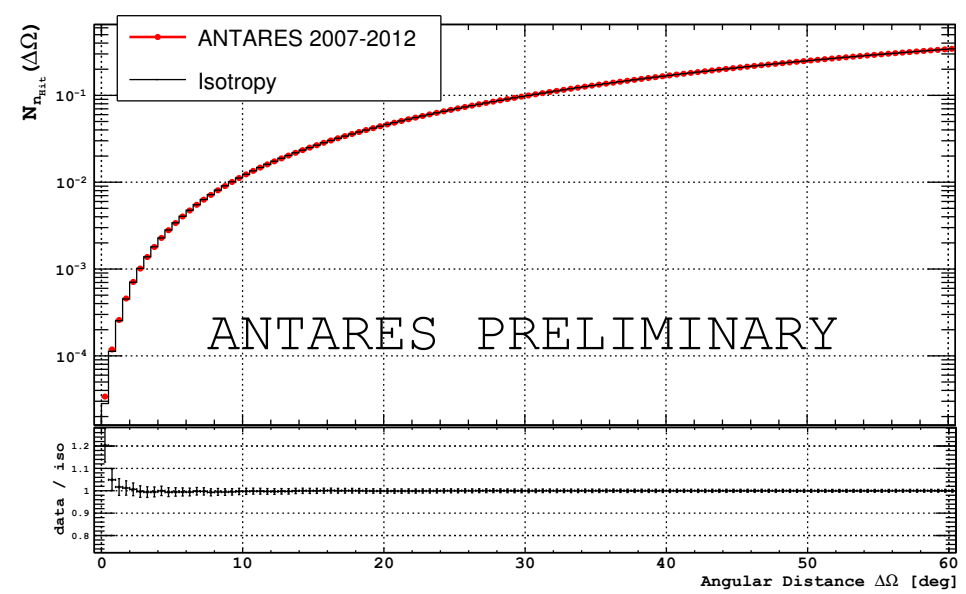

Figure 2: Cumulative autocorrelation distributions for the 2007-2012 ANTARES data set (red points) and for the average isotropic ensemble (black line).

simulated population of sources inspired by [5], whose mean neutrino luminosities follow a power law

$$
\frac{d N}{d S} \propto \cdot S^{-\gamma}
$$

between some limits $S_{\min }$ and $S_{\max }$, where $S$ is a dimensionless quantity corresponding to the integrated mean luminosity expressed in number of detected neutrinos. $S_{\max }$ has been fixed to the faintest point source compatible with the limit set by the search for point sources with ANTARES data [3], and $S_{\min }$ is a free parameter bounded above by $S_{\max }$, which will characterize a source population.

The luminosity function can be considered as a proxy of the power of the sources. Although the luminosity function for neutrino sources is unknown, one can assume that it follows the same general rules as the luminosity functions in x-rays or gamma rays. We can thus rely on known populations of point sources to choose the range of $\gamma$. Studies of galactic type sources like lowmass X-ray binaries in Centaurus A [6] [7] or in the Milky Way [9] yield a typical spectral index below 2.0, and Fermi LAT studies show that the gamma ray luminosity function of AGNs is well described by a power law with $\gamma \sim 2.2$ [8].

Therefore a signal sky map is characterized by the couple of parameters, $\left(\gamma, S_{\min }\right)$ and the proportion of injected signal events. $\gamma$ characterizes the type of objects that constitute the population. It drives the relative contributions of the different source luminosities to the total flux. For a given average detected flux, populations characterized by higher values of $\gamma$ will consist in a higher number of less luminous sources. $S_{\min }$, independently of the type of sources, will characterize their effective average minimal detectable luminosity within the population. We will test the parameter space $1.8<\gamma<2.3$, and $0.025<S_{\min }<1$.

The energy estimator of the signal events is generated from Monte Carlo simulations assuming that the signal events follow an $E^{-2}$ spectrum. Figure 3 shows an example of a sky map where the $0.5 \%$ of the background events have been replaced by events coming from sources distributed with a spectral index of $\gamma=1.9$ and $S_{\min }=0.025$ 


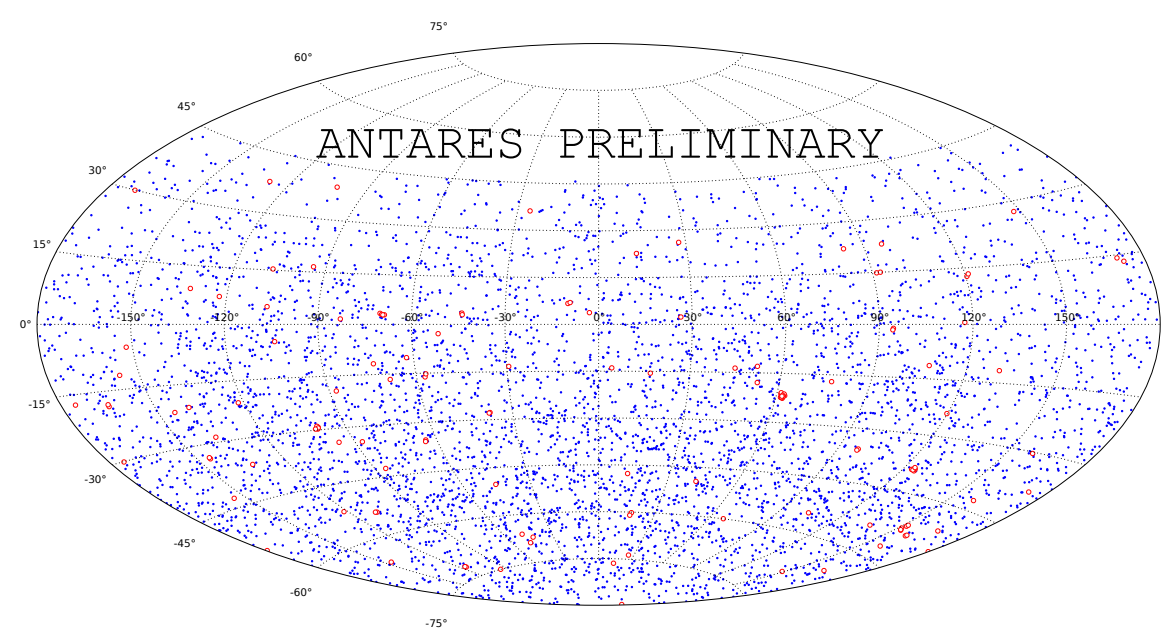

Figure 3: Example of a sky map with signal coming from unresolved point sources. The events in blue come from background while the red events correspond to signal.

\subsubsection{Discovery potential of a population of unresolved point sources}

Following the autocorrelation method described in section 2.1, sky maps characterized by different couples $\left(\gamma, S_{\min }\right)$ can be analysed to determine the cumulative diffuse flux of cosmic neutrinos coming from populations of unresolved point sources that would be detected at a $3 \sigma$ significance with a $90 \%$ probability. As the neutrino luminosity function for different kinds of sources is unknown, the spectral index was assumed to lay within the same range as the spectral index for the luminosity function in x-rays or in gamma rays for different kinds of sources [5]. The discovery potential as a function of the spectral index $\gamma$ and $S_{\min }$ under the above conditions is shown in fig.4

\subsection{Results and Discussion}

The autocorrelation method has been applied to the 5243 events measured by ANTARES from 2007 to 2012 and the result has been compared to the one expected from a purely isotropic sample. The comparison, showed that the largest difference between the distributions corresponds to an excess of the ANTARES neutrino candidates with respect to the isotropic ensemble, at scales $<0.5^{\circ}$. The statistical analysis leads to the conclusion that this corresponds to a $\sim 2.3 \sigma$ excess. In previous point source search analyses [2] [3] a $2.2 \sigma$ excess was found around $(\alpha, \delta)=(313.20,-$ 64.90). Removing events closer than $0.5^{\circ}$ from this point reduces the significance of the observed excess in the current analysis to $2.15 \sigma$, and therefore we can conclude that they are not responsible for the observed deviation with respect to the background.

We thus set upper limits on the cumulative diffuse flux of the model of unresolved point sources population presented above, as a function of the spectral index $\gamma$ and $S_{\min }$, which are presented in figure 5. As expected from the definitions in section 2.5.1, the constraints are stronger for increasing $S_{\min }$. One can also see that populations of sources with low values of $\gamma$ (similar to 


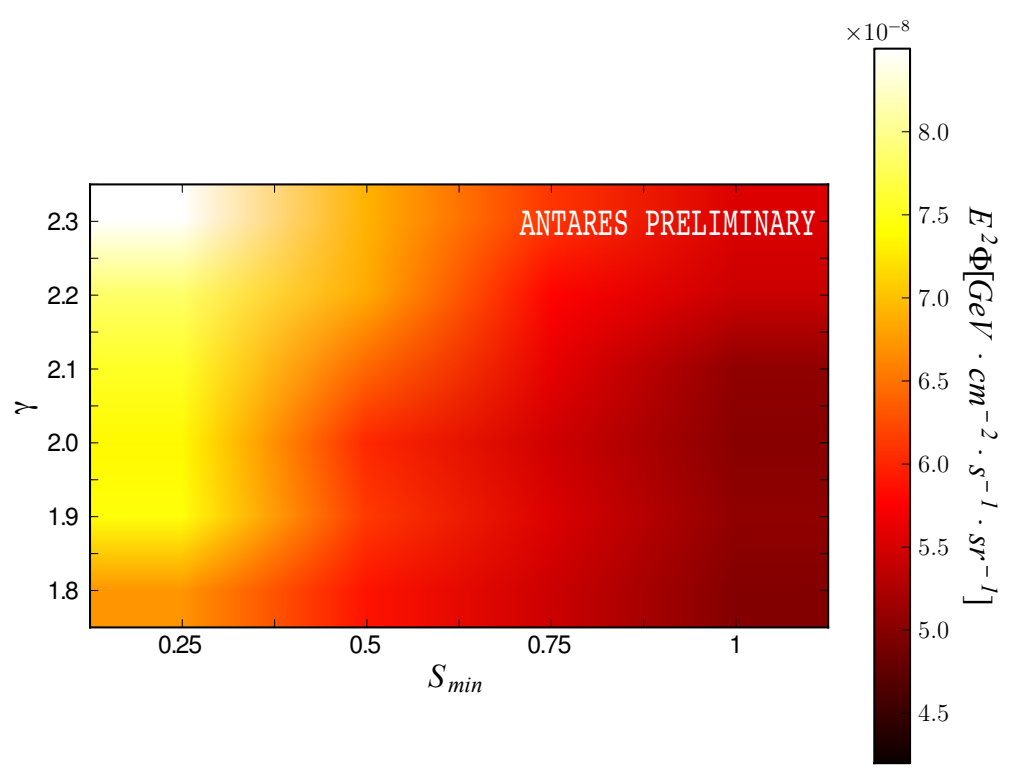

Figure 4: Discovery potential at $3 \sigma$ significance with a $90 \%$ probability of the model presented in section 2.5.1 as a function of the model's parameters $\left(\gamma, S_{\min }\right)$.

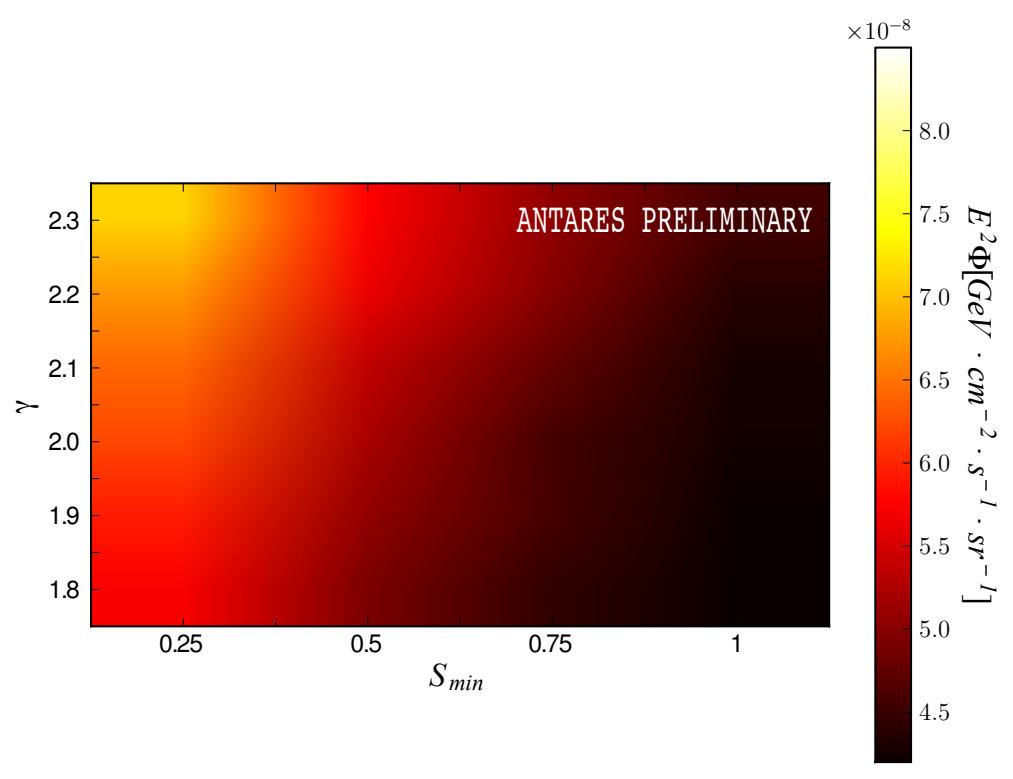

Figure 5: $90 \%$ confidence level upper limit on the cumulative diffuse flux as a function of the model's parameters $\left(\gamma, S_{\min }\right)$. 
high energy galactic type sources as x-ray binaries) are more strongly constrained. These results, in addition and independently of the high visibility of the galactic region, confirm the latter as a favoured subject of study for the ANTARES neutrino telescope.

\section{Summary}

The detection of astrophysical neutrinos would provide fundamental information about the location of CR sources. This is the aim of the ANTARES neutrino telescope. In this analysis an improved autocorrelation method was applied in order to search for clustering in the directions of the reconstructed neutrino candidates but not significant deviation from background was found. Upper limits for a neutrino flux coming from a population of point sources characterized by a two parameter model were set.

\section{References}

[1] J.A. Aguilar et al. ANTARES: the first undersea neutrino telescope, Nuclear Inst. and Methods in Physics Research, A 656 (2011) pp. 11-38

[2] S. Adrian-Martinez et al. Searches for clustering in the time integrated skymap of the ANTARES neutrino telescope, JCAP05 (2014)0001

[3] S. Adrian-Martinez et al. Search for cosmic neutrino point sources with four year data of the ANTARES telescope, The Astrophysical Journal 760:53(2012)

[4] ANTARES Collaboration, 2pt correlation analysis of ANTARES data (ICRC 2013, Rio de Janeiro), arXiv:1312.4308 [astro-ph.HE].

[5] Tracy R. Slatyer, Douglas P. Finkbeiner A statistical test of emission from unresolved point sources, Mon. Not. R. Astron. Soc. 405, 1777-1786 (2010)

[6] Voss R., et al., Luminosity functions of LMXBs in Centaurus A: globular clusters versus the field, The Astrophysical Journal ,701:471-48

[7] R. Voss, M. Gilfanov The Luminosity Function of X-ray Point Sources in Centaurus A, Astron.Astrophys. 447 (2006) 71

[8] M. Ackermann et al. [Fermi-LAT Collaboration], The Third Catalog of Active Galactic Nuclei Detected by the Fermi Large Area Telescope, arXiv:1501.06054 [astro-ph.HE].

[9] M. Gilfanov, Low mass x-ray binaries as a stellar mass indicator of the host galaxy, Mon. Not. Roy. Astron. Soc. 349 (2004) 146 [astro-ph/0309454]. 